\title{
INFLUÊNCIA DO TRATAMENTO TÉRMICO NA RESISTÊNCIA AO DESGASTE DE REVESTIMENTOS DE NÍQUEL QUÍMICO COM ALTO TEOR DE FÓSFORO
}

Felipe Samuel Goettems ' Jane Zoppas Ferreira'

\section{Resumo}

No presente trabalho foi avaliada a resistência ao desgaste de revestimentos de níquel químico com alto teor de fósforo (9-10\% P) após passar por três diferentes condições de tratamento térmico, incluindo a condição de como depositado, ou seja, sem tratamento posterior. Os tratamentos foram realizados nas temperaturas de $320^{\circ} \mathrm{C}, 400^{\circ} \mathrm{C} \mathrm{e} 500^{\circ} \mathrm{C}$ em diferentes tempos, sendo estas condições escolhidas com base no resultado do ensaio de calorimetria exploratória diferencial (DSC). Feito isso as amostras foram então caracterizadas através do ensaio de desgaste do tipo "ball on plate", para depois suas trilhas de desgaste serem avaliadas em microscópio. Ao final, chegou-se à conclusão de que o tratamento térmico afeta de forma positiva a resistência ao desgaste dos depósitos de Ni-P, tendo como $320^{\circ} \mathrm{C}$ por 9 horas e $400^{\circ} \mathrm{C}$ por I hora as condições que apresentaram o melhor resultado. Este fato se deve a transformação de sua estrutura amorfa para uma estrutura cristalina de níquel com precipitados de $\mathrm{Ni}_{3} \mathrm{P}$ ocorrida entre $320^{\circ} \mathrm{C}$ e $360^{\circ} \mathrm{C}$, verificada através da difração de raios-X (DRX).

Palavras-chave: Revestimento metálico; Resistência ao desgaste; Níquel químico; Tratamento térmico.

\section{EFFECT OF HEAT TREATMENT ON THE WEAR BEHAVIOUR OF ELECTROLESS HIGH PHOSPHORUS NICKEL COATING}

\begin{abstract}
In this present work was studied the influence of heat treatment on the wear resistance of electroless high phosphorus nickel coating (9-10\% P wt.). Three different treatment conditions as well as untreated condition were evaluated at temperatures of $320^{\circ} \mathrm{C}, 400^{\circ} \mathrm{C}$ and $500^{\circ} \mathrm{C}$ under different holding times. The selection of the heat treatment conditions was chosen considering the result obtained by a differential scanning calorimetry (DSC) analysis. The wear behaviour of the Ni-P samples was investigated through a non-lubricated ball on plate test and the results showed that the heat treatment affect positively the wear resistance of Ni-P coatings. Treatment conditions of $320^{\circ} \mathrm{C}$ with 9 hours holding time and $400^{\circ} \mathrm{C}$ with I hour holding time showed the best results due to a structural change from amorphous supersatured solid solution of phosphorus in nickel to a crystalline structure of nickel crystallites and nickel phosphides $\left(\mathrm{Ni}_{3} \mathrm{P}\right)$ occurred between $320^{\circ} \mathrm{C}$ and $360^{\circ} \mathrm{C}$ verified after X-ray analysis.
\end{abstract}

Keywords: Metallic coatings; Wear resistance; Nickel electroless; Heat treatment.

\section{INTRODUÇÃO}

Nas últimas décadas houve um crescente aumento na busca por revestimentos metálicos com aplicação na engenharia capazes de apresentar boas propriedades mecânicas em situações severas de aplicação, incluindo-se neste grupo de propriedades a resistência ao desgaste, permitindo que seja possível aliar características especificas do substrato com outras em que só seja possível atingir através da aplicação de outro material.
Um tipo de revestimento que está recebendo atenção especial nos últimos anos devido à sua grande versatilidade é o níquel químico, que vem ganhando mais e mais espaço em diversos setores da indústria tais como automotiva, alimentícia, extrativa, óleo e gás, etc. Este depósito possui uma grande vantagem em relação aos demais revestimentos metálicos usualmente utilizados, cromo duro eletrodepositado por exemplo, justamente pelo fato de não utilizar corrente

'Programa de Pós-graduação em Engenharia de Minas, Metalúrgica e de Materiais - PPGE3M, Laboratório de Corrosão, Proteção e Reciclagem de Materiais - LACOR, Universidade Federal do Rio Grande do Sul - UFRGS, Porto Alegre, RS, Brasil. E-mail: felipegoettems@yahoo.com.br 
elétrica para sua obtenção, eliminando assim diversos problemas decorrentes da eletricidade, a citar: eliminação da formação de trincas na superfície (formada devido a liberação de $\mathrm{H}_{2}$ durante a eletrodeposição) e recobrimento mais uniforme e menos rugoso (não sofre do efeito das pontas ocasionado pela concentração de cargas elétricas) [I-3].

Até pouco tempo atrás, revestimentos de níquel químico com alto teor de fósforo (acima de $9 \%$ em peso de $\mathrm{P}$ ) eram amplamente utilizados onde se necessitava ótima resistência à corrosão devido à sua ótima performance neste quesito $\mathrm{e}$ ancorado pela sua reconhecida característica amorfa [4-7], ao contrário de revestimentos com baixo teor de fósforo e sua estrutura nanocristalina. Porém, estudos mais recentes remetem para aplicação de revestimentos de $\mathrm{Ni}$ - $\mathrm{P}$ com alto teor de fósforo em aplicações onde se requeira alta resistência ao desgaste, devido à uma característica importante dos depósitos de níquel químico: a capacidade de alterar suas propriedades através de tratamento térmico [8-10].

Já é conhecido que o tratamento térmico em temperaturas próximas a $340^{\circ} \mathrm{C}$ faz com que depósitos de Ni-P deixem de ser amorfos e passem a ser cristalinos através da precipitação de fosfeto de níquel $\left(\mathrm{Ni}_{3} \mathrm{P}\right)$ e da formação de cristalitos de $\mathrm{Ni}$ com estrutura cúbica de face centrada $[6,1 \mathrm{I}, 12]$. Aliado a isto, um estudo realizado por Taheri [13] mostrou que a precipitação de fosfetos e de cristalitos de Ni é tão maior quanto maior o teor de fósforo na liga $\mathrm{Ni}$-P, levando a uma transformação estrutural completa de amorfo para cristalino na condição de tratamento térmico de $400^{\circ} \mathrm{C}$ por I hora, e consequentemente aumentando sua dureza e resistência ao desgaste.

Estudos também já demostraram que tratamentos térmicos em temperaturas superiores a $450^{\circ} \mathrm{C}$ ocasionam um efeito deletério na resistência ao desgaste de revestimentos de Ni-P, devido principalmente ao crescimento de grão do material e pela formação de compostos intermetálicos indesejáveis na interface substrato/revestimento [14,15], e que tratamentos em temperaturas inferiores a $300^{\circ} \mathrm{C}$ não atuam de modo positivo na resistência ao desgaste, muito devido a não chegada na janela de temperaturas responsáveis pela transformação de fases em revestimentos de Ni-P $[8,15]$.

Neste estudo, um substrato de aço inoxidável martensítico SAE HNV3 foi revestido com uma liga de níquel químico com alto teor de fósforo. Foram aplicados tratamentos térmicos no depósito em diferentes condições e sua resistência ao desgaste fora avaliada através de um ensaio "ball on plate" sem lubrificação comparando-se ao revestimento sem tratamento, procedido por análise de calorimetria exploratória diferencial (DSC), difração de raios-X (DRX), espectroscopia de energia dispersiva (MEV/EDS), medição de rugosidade e espessura dos depósitos. Os tratamentos térmicos nas temperaturas de $320^{\circ} \mathrm{C}$, ou seja, muito próxima da temperatura de transição microestrutural, $400^{\circ} \mathrm{C}$, que teoricamente proporciona uma completa transformação amorfa/cristalina, e $500^{\circ} \mathrm{C}$, na qual é superior ao limite considerado ideal em termos de resistência ao desgaste, foram propositalmente escolhidos justamente no intuito de se comprovar tais fenômenos supracitados.

Foi avaliada a relação que o tratamento térmico possui na resistência ao desgaste dos revestimentos de $\mathrm{Ni}-\mathrm{P}$, bem como a explicação por tal fenômeno ocorrer através dos ensaios complementares e trabalhos já publicados na literatura.

\section{MATERIAIS E MÉTODOS}

O substrato utilizado para a deposição do níquel químico foi uma barra cilíndrica retificada de $9,5 \mathrm{~mm}$ de diâmetro e fabricada com um aço inoxidável martensítico SAE HNV3. Antes da deposição, as amostras passaram por um desengraxe realizado em duas etapas: químico, através de desengraxante comercial Uniclean 169 durante 10 minutos e eletrolítico, através de desengraxante Uniclean 264 durante I minuto. Para aumentar a adesão, as amostras sofreram ainda uma ativação superficial com $\mathrm{H}_{2} \mathrm{SO}_{4} 5 \%$ durante 2 minutos. A deposição de Ni-P ocorreu em um banho ácido com $\mathrm{pH} 4,8$, temperatura de $88^{\circ} \mathrm{C}$, teor de níquel de 5,6 g/L e carga do tanque de $1,25 \mathrm{dm}^{2} / \mathrm{L}$. As amostras permaneceram imersas no banho durante um período de 25 minutos. Para a realização dos tratamentos térmicos, as amostras foram conduzidas a um forno elétrico sem atmosfera controlada de modo a obter as seguintes condições de tratamento, além da condição sem tratamento algum ( $\left(\mathrm{EN} \mathrm{S} / \mathrm{T}^{\circ} \mathrm{T}^{\circ}\right): 320^{\circ} \mathrm{C}$ por 9 horas (EN 320-9), $400^{\circ} \mathrm{C}$ por I hora (EN $400-\mathrm{I}$ ) e $500^{\circ} \mathrm{C}$ por I hora (EN 500-I). O tempo maior de 9 horas para a amostra tratada em $320^{\circ} \mathrm{C}$ foi propositalmente ajustado para permitir que haja uma completa transformação de fases, visto que a temperatura está próxima do limite de transição cristalina e I hora talvez não seria suficiente para garantir total transformação.

Para a verificação do teor de fósforo nos revestimentos de Ni-P, foi utilizada a técnica de Espectroscopia de Energia Dispersiva (EDS) através de um Microscópio Eletrônico de Varredura (MEV) Phenon Pro-X. A espessura dos depósitos foi medida utilizando-se um Microscópio Óptico (MO) Olympus após corte em secção transversal das peças, embutimento e preparação metalográfica padrão. A rugosidade dos revestimentos foi medida através do rugosímetro portátil Digimess 400.200 , sendo a área de varredura utilizada pelo equipamento de $5 \times 0,8 \mathrm{~mm}$. O ensaio de Calorimetria Exploratória Diferencial (DSC) foi realizado através do aparelho Netzsch Pegasus $404 \mathrm{FI}$, em um intervalo de temperaturas de $20^{\circ} \mathrm{C}$ até $500^{\circ} \mathrm{C}$, com uma taxa de aquecimento de $10 \mathrm{~K} / \mathrm{s} \mathrm{e}$ atmosfera controlada de $\mathrm{N}_{2} / \mathrm{O}_{2}$. A análise de Difração de Raios-X (DRX) foi conduzida através do aparelho Philips $X$ 'Pert em um intervalo $2 \theta$ de $5^{\circ}$ até $75^{\circ}$. Ambos ensaios de DSC e DRX foram realizados nas amostras no estado de pó metálico após uma raspagem mecânica realizada nas peças depositadas. O tratamento dos resultados obtidos por DRX foram feitos utilizando-se software específico para cristalografia X'Pert High Score Plus. 
Para a avaliação da resistência ao desgaste foi utilizado um tribômetro com deslocamento linear CETR, sem lubrificação, utilizando-se como contra-face uma esfera de alumina de $4,72 \mathrm{~mm}$ de diâmetro, força de ensaio de $6 \mathrm{~N}$, frequência de $3 \mathrm{~Hz}$, tamanho linear da trilha de $2 \mathrm{~mm}$ e tempo de ensaio de 40 minutos. Após os testes de desgaste, as amostras passaram por uma avaliação em Microscópio Óptico.

\section{RESULTADOS E DISCUSSÃO}

\section{I Avaliação Física e Microestrutural}

Na Tabela I estão representados o teor de fósforo encontrado para cada amostra bem como a espessura do revestimento.

Observa-se que, considerando o desvio padrão das medições de cada amostra, os resultados são próximos e não devem representar diferenças nos resultados dos outros ensaios. Além disso, todas as amostras possuem teores de fósforo superiores a $9 \%$ em peso, representando, segundo a literatura $[16,17]$, como sendo um revestimento de níquel químico de alto fósforo. Com relação a espessura dos revestimentos, nota-se que todas as amostras estão muito próximas uma das outras, não representando diferenças que possam interferir nos ensaios posteriores.

As condições de tratamento térmico foram escolhidas seguindo o caminho aberto pela literatura $[4,8,9]$ como sendo mais eficientes no sentido de aumentar as propriedades dos revestimentos de Ni-P. Partindo-se desta ideia inicial, foi realizado um ensaio de DSC para verificar com maior precisão a faixa de temperaturas em que ocorre a modificação estrutural para o tipo de revestimento estudado neste trabalho, ou seja, para este específico teor de fósforo. Na Figura I estão os resultados obtidos após análise de DSC para uma amostra depositada sem tratamento térmico.

Nota-se que há um pico exotérmico na faixa de $320-360^{\circ} \mathrm{C}$. Este resultado vai ao encontro da literatura $[6,13,18]$ que descreve uma transformação estrutural de amorfo ou nano cristalino para uma estrutura totalmente cristalina em temperaturas entre $300^{\circ} \mathrm{C} \mathrm{e} 400^{\circ} \mathrm{C}$ para revestimentos de Ni-P.

A confirmação desta transformação foi verificada através de análises de DRX. Na Figura 2 é apresentado o resultado obtido na difração de raios-X para a amostra sem

Tabela I. Teor de fósforo e espessura dos revestimentos após deposição

\begin{tabular}{ccc}
\hline Amostra & Teor de Fósforo* & Espessura do Depósito* \\
\hline EN S/T० ${ }^{\circ}$ & $9,4 \% \pm 0,5$ & $5,7 \mu \mathrm{m} \pm 0,3 \mu \mathrm{m}$ \\
EN $320-9$ & $9,1 \% \pm 0,5$ & $6,2 \mu \mathrm{m} \pm 0,2 \mu \mathrm{m}$ \\
EN $400-1$ & $10,1 \% \pm 0,5$ & $5,2 \mu \mathrm{m} \pm 0,4 \mu \mathrm{m}$ \\
EN 500- 1 & $9,9 \% \pm 0,5$ & $6,4 \mu \mathrm{m} \pm 0,3 \mu \mathrm{m}$ \\
\hline
\end{tabular}

*Média obtida após quatro medições. tratamento térmico em comparação com amostras após tratamento térmico. Nota-se claramente um único pico difuso por volta do ângulo $2 \theta=45^{\circ}$ para a amostra sem tratamento térmico correspondente a solução supersaturada de fósforo em níquel e caracterizando o caráter amorfo. Já para as amostras com tratamento térmico, verifica-se que houve uma cristalização, ou seja, uma transformação de uma solução supersaturada de fósforo em níquel para precipitados de fosfeto de níquel $\left(\mathrm{Ni}_{3} \mathrm{P}\right)$ e cristalitos de $\mathrm{Ni}$ (cúbico de face centrado, planos (III) e (220)). Esta característica já era esperada e reflete bem o que foi pesquisado até $o$ atual momento para o tratamento térmico de revestimentos de níquel fósforo [4,8,19-21].

\subsection{Avaliação Tribológica}

Considerando que os resultados de desgaste são fortemente influenciados pela condição superficial das peças em contato mútuo, a rugosidade média das amostras foi medida através de um rugosímetro. Os resultados podem ser vistos na Tabela 2 abaixo. Verifica-se que, em alguns casos, até mesmo houve uma redução da rugosidade em comparação com o substrato no qual foram depositadas, isto pode estar associado ao fato de que o revestimento de $\mathrm{Ni}-\mathrm{P}$ faz uma cobertura homogênea da superfície da peça, fazendo com que regiões mais rugosas sejam recobertas pelo depósito. Este fato já foi observado anteriormente por Lee [22] e Vitry [23].

$\mathrm{Na}$ Figura 3 estão representadas as curvas que relacionam o coeficiente de atrito (COF) ao longo do tempo obtidas após o ensaio de desgaste em condição não lubrificada das amostras de Ni-P e uma contra-face de alumina.

Observa-se que o maior coeficiente de atrito foi verificado para a amostra $\mathrm{EN} S / \mathrm{T}^{\circ} \mathrm{T}^{\circ}$, seguido pela amostra EN 500-I. Os menores valores de COF foram verificados para os tratamentos realizados em $400^{\circ} \mathrm{C} \mathrm{e} 320^{\circ} \mathrm{C}$, amostras

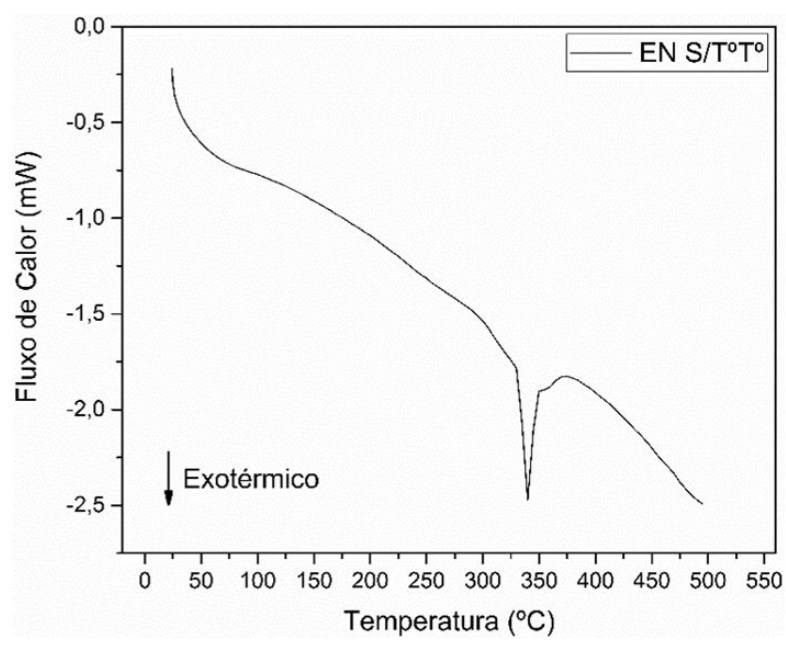

Figura I. Curva DSC para a amostra com revestimento Ni-P sem tratamento térmico. 

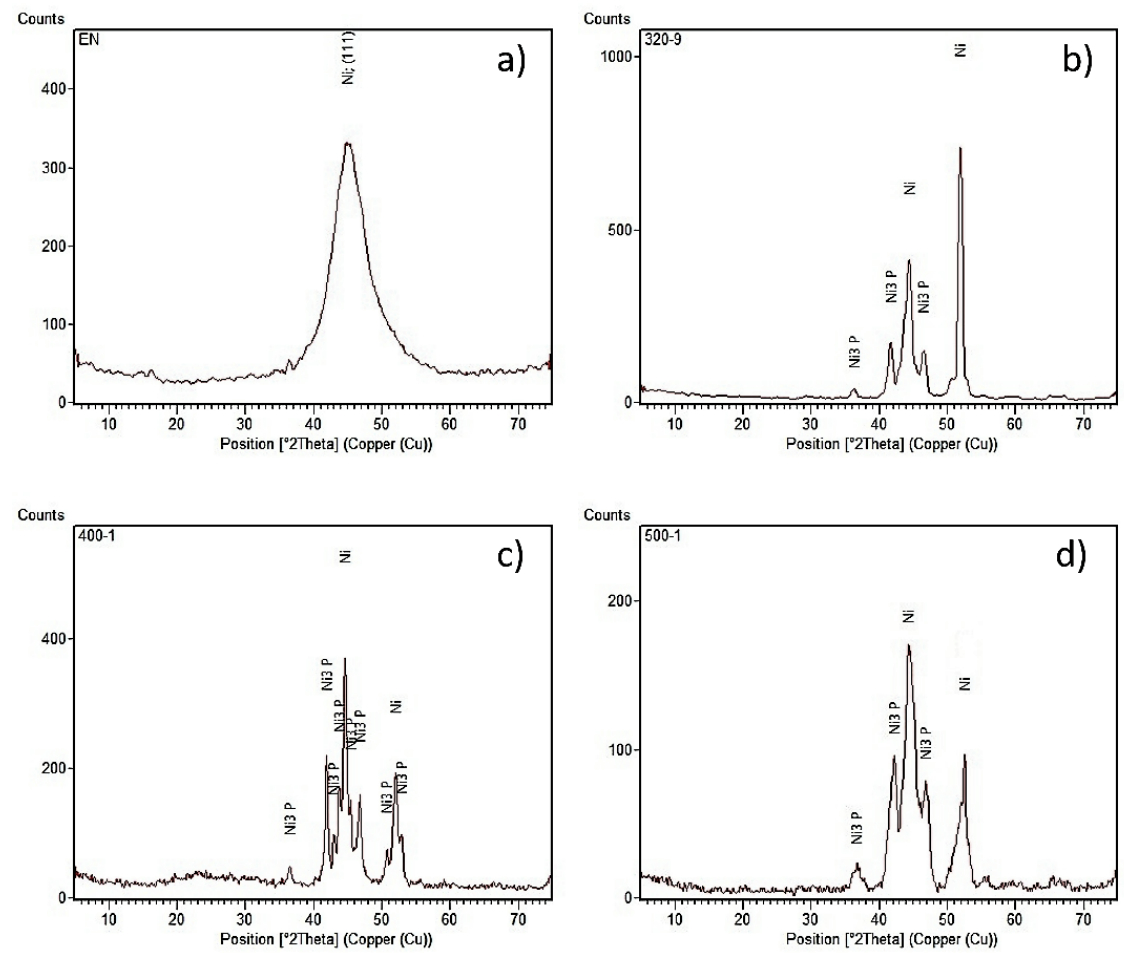

Figura 2. Análise de DRX para as amostras revestidas de Ni-P nas seguintes condições: (a) EN S/Tº'; (b) EN 320-9; (c) EN 400-I; (d) EN 500-I .

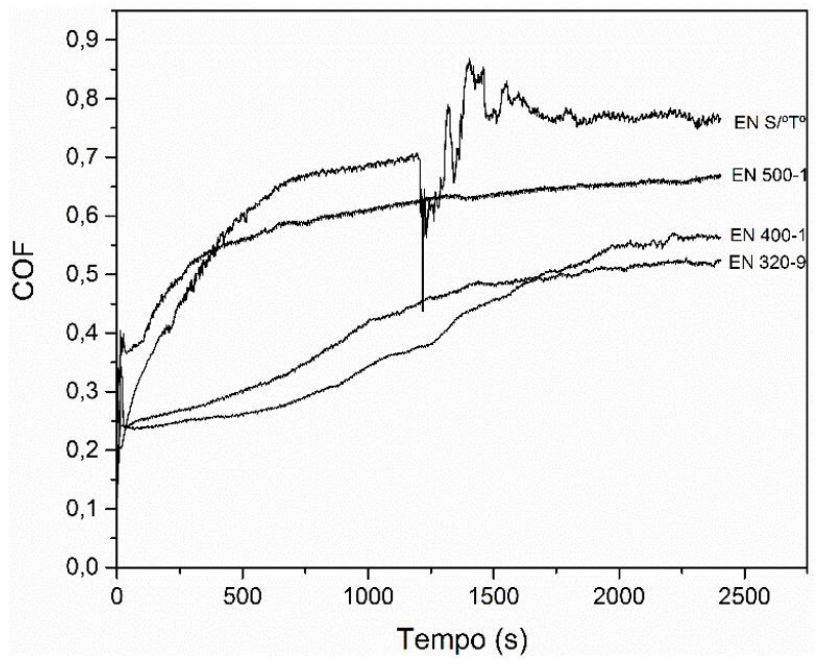

Figura 3. Curvas de COF ao longo do tempo para as amostras revestidas com $\mathrm{Ni}$-P.

Tabela 2. Rugosidade média obtida para as amostras

\begin{tabular}{cc}
\hline Amostra & *Rugosidade Média $\mathbf{R}_{\mathrm{a}}$ \\
\hline Substrato & $0,24 \mu \mathrm{m} \pm 0,03 \mu \mathrm{m}$ \\
EN S/T'T $T^{\circ}$ & $0,17 \mu \mathrm{m} \pm 0,03 \mu \mathrm{m}$ \\
EN $320-9$ & $0,19 \mu \mathrm{m} \pm 0,02 \mu \mathrm{m}$ \\
EN $400-1$ & $0,23 \mu \mathrm{m} \pm 0,01 \mu \mathrm{m}$ \\
EN $500-$ I & $0,22 \mu \mathrm{m} \pm 0,01 \mu \mathrm{m}$ \\
\hline
\end{tabular}

* Média obtida após quatro medições.
EN 320-9 e EN 400-I, indicando estas temperaturas mais indicadas em se tratando de resistência ao desgaste. Esta diminuição do COF para revestimentos de Ni-P após o tratamento térmico provavelmente está associada com a formação de uma fina camada de óxidos na superfície devido ao aquecimento durante o tratamento. Estes óxidos agiriam como lubrificantes naturais, favorecendo a diminuição do COF [14]. Importante ressaltar que esta formação de óxidos só foi evidenciada quando o tratamento térmico é realizado em atmosfera não controlada, ou seja, com oxigênio abundante $[4,8]$.

Para a amostra de Ni-P EN S/T $T^{\circ} T^{\circ}$ observa-se que no início do ensaio o coeficiente de atrito gira em torno de 0,2 , valor este conhecido para revestimentos de Ni-P [24]. Com o passar do tempo, o valor de COF começa a subir gradualmente até ocorrer a quebra do filme, por volta de 1250 segundos de ensaio, evidenciado por uma queda brusca do COF. Após isso, o COF assume valores maiores e constantes da ordem de 0,8 até o final do ensaio, provavelmente alcançando o substrato. Analisando-se os resultados do coeficiente de atrito para a amostra EN 320-9, nota-se que o valor de COF é novamente coerente com revestimentos de Ni-P e inicia-se em torno de 0,2. Há um aumento gradual, porém, menos acentuado do COF em comparação com a amostra sem tratamento térmico, e não se percebe ainda a quebra por completo do filme. Após o final do ensaio, a amostra EN 320-9 apresentou um coeficiente de atrito relativamente baixo da ordem de 0,5 . O comportamento do COF para a amostra EN 400-I é muito similar ao da 
amostra EN 320-9, iniciando-se em valores da ordem de 0,2 e aumentando gradual e lentamente até valores finais um pouco maiores que a amostra anterior, em torno de 0,55 . Porém, da mesma maneira não foi observado a ruptura do filme e a chegada do COF a valores próximos a amostra sem tratamento térmico. Já o comportamento do coeficiente de fricção para a amostra EN 500-I foi diferente dos dois outros após tratamento térmico. O COF apresentou um rápido aumento para 0,4 logo nos primeiros momentos do ensaio, aumentando gradualmente até chegar ao valor de 0,65 . Porém, da mesma maneira ainda não chegou aos valores do coeficiente de atrito da amostra sem tratamento, aparentemente não rompendo o filme.

Analisando-se, para a amostra EN S/ $/ \mathrm{T}^{\circ} \mathrm{T}^{\circ}$ as trilhas deixadas pelo ensaio de desgaste em microscópio óptico, notam-se claramente estrias típicas de desgaste abrasivo, tanto na peça (Figura 4b) como na contra-face (Figura 4c). Estas estrias são bem visíveis e em grande quantidade, pois formam um ângulo de $90^{\circ} \mathrm{com}$ as marcas de usinagem da peça. É possível notar também material aderido na contra-face, sinal típico de desgaste adesivo. Estes dois mecanismos de desgaste encontrados apenas corroboram para o resultado que já era esperado em se tratando de materiais metálicos [25]. Também na Figura $4 b$ verifica-se que a largura da trilha, ou seja, o diâmetro que a esfera de alumina conseguiu penetrar na amostra durante o ensaio, é de $463 \mu \mathrm{m}$.

Analisando-se as imagens da Figura 5, verifica-se que a amostra EN 320-9 também possui os dois mecanismos de desgaste típicos de materiais metálicos: abrasivo, evidenciado pelas estrias encontradas (Figura 5a) e adesivo, pelo material aderido na contra-face (Figura 5c). A quantidade de estrias verificada é bem menos evidente que para o caso do revestimento sem tratamento térmico, e o tamanho da trilha foi medido como sendo da ordem de $168 \mu \mathrm{m}$, bem menor se comparado a amostra $\mathrm{EN} \mathrm{S} / \mathrm{T}^{\circ} \mathrm{T}^{\circ}$.

Da mesma maneira que as amostras anteriores, a condição chamada de EN 400-I apresentou os dois mecanismos de desgaste, abrasivo (nível de estrias semelhante a amostra EN 320-9) e adesivo, conforme mostra a Figura 6. O tamanho da trilha foi medido em torno de $164 \mu \mathrm{m}$, muito semelhante a amostra EN 320-9, o que é condizente com o verificado nas curvas de COF ao longo do tempo apresentadas na Figura 3.

A trilha de desgaste da amostra EN 500-I apresentou uma largura de $204 \mu \mathrm{m}$, onde foi possível observar os dois mecanismos de desgaste novamente, abrasivo (verificado pela presença de estrias, Figura 7a) e adesivo (verificado pela presença de material aderido na contra-face, Figura 7c).

O melhor comportamento de desgaste verificado pelo menor tamanho das trilhas para as amostras de Ni-P após tratamento térmico já era de certa forma esperado. Diversos estudos já foram feitos neste sentido, e todos reportam que a transformação da fase metaestável de fósforo saturado em uma matriz de níquel para as fases esperadas no diagrama de equilíbrio, fosfetos e cristalitos de níquel, é a grande responsável pelo aumento da dureza dos revestimentos, e consequentemente pelo aumento da resistência ao desgaste $[4,5,8,9]$. A queda na resistência ao desgaste da amostra EN 500-I em relação as demais condições de tratamento térmico também já foi citada [8], e está provavelmente relacionada ao aumento do tamanho dos cristalitos e fosfetos presentes na microestrutura, ocasionado pela maior temperatura de tratamento térmico e pela difusão de elementos provenientes do substrato para
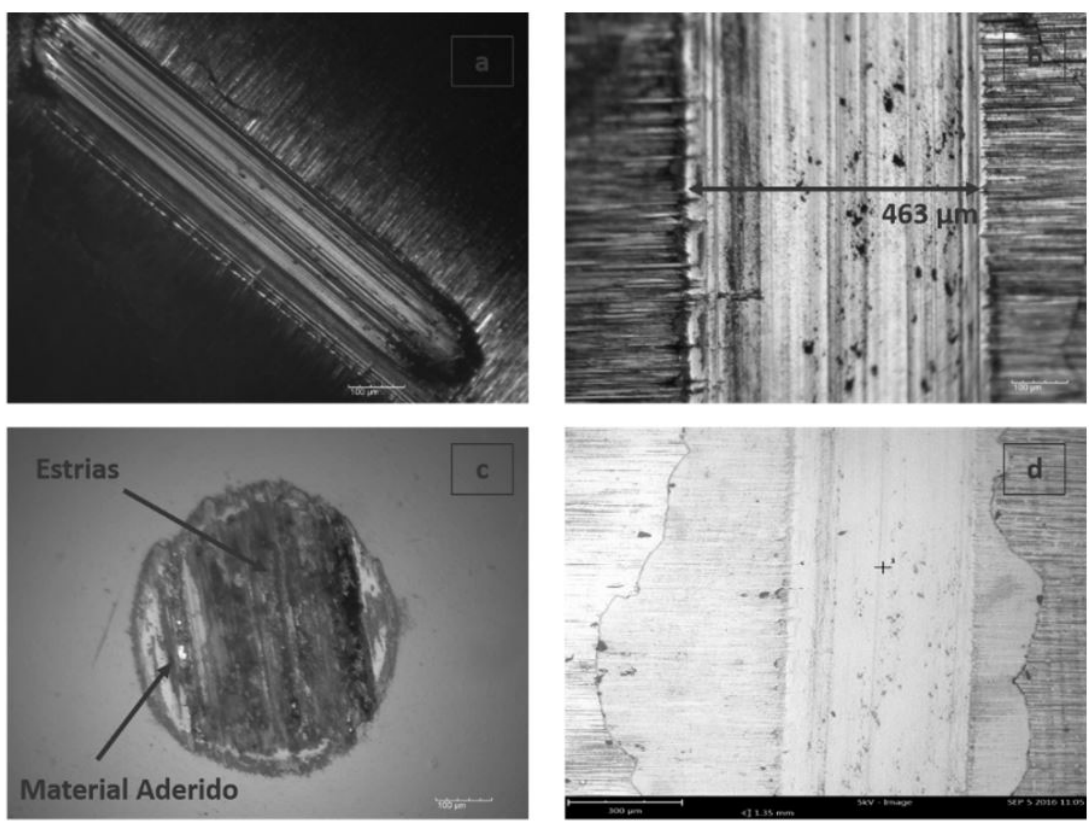

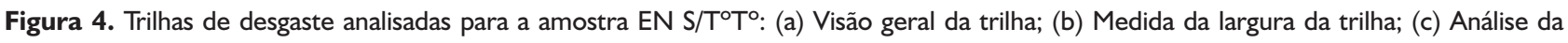
contra-face; (d) Imagem ampliada da trilha. 

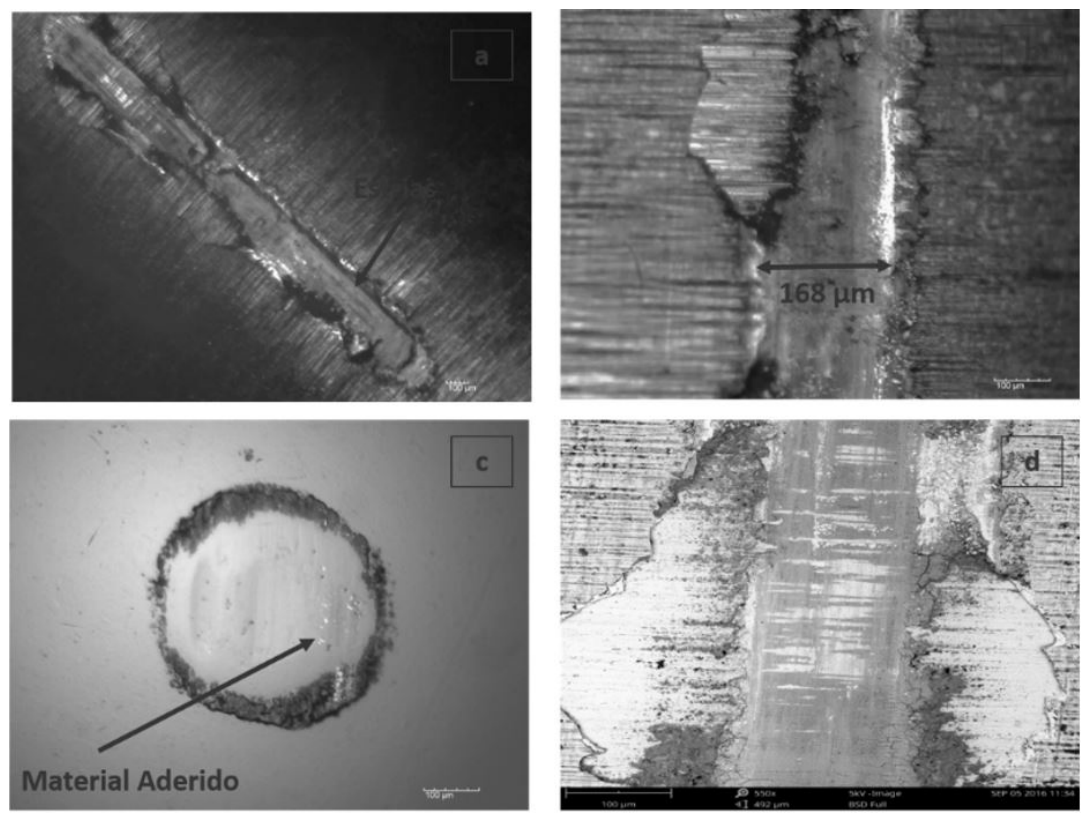

Figura 5. Trilhas de desgaste analisadas para a amostra EN 320-9: (a) Visão geral da trilha; (b) Medida da largura da trilha; (c) Análise da contra-face; (d) Imagem ampliada da trilha.
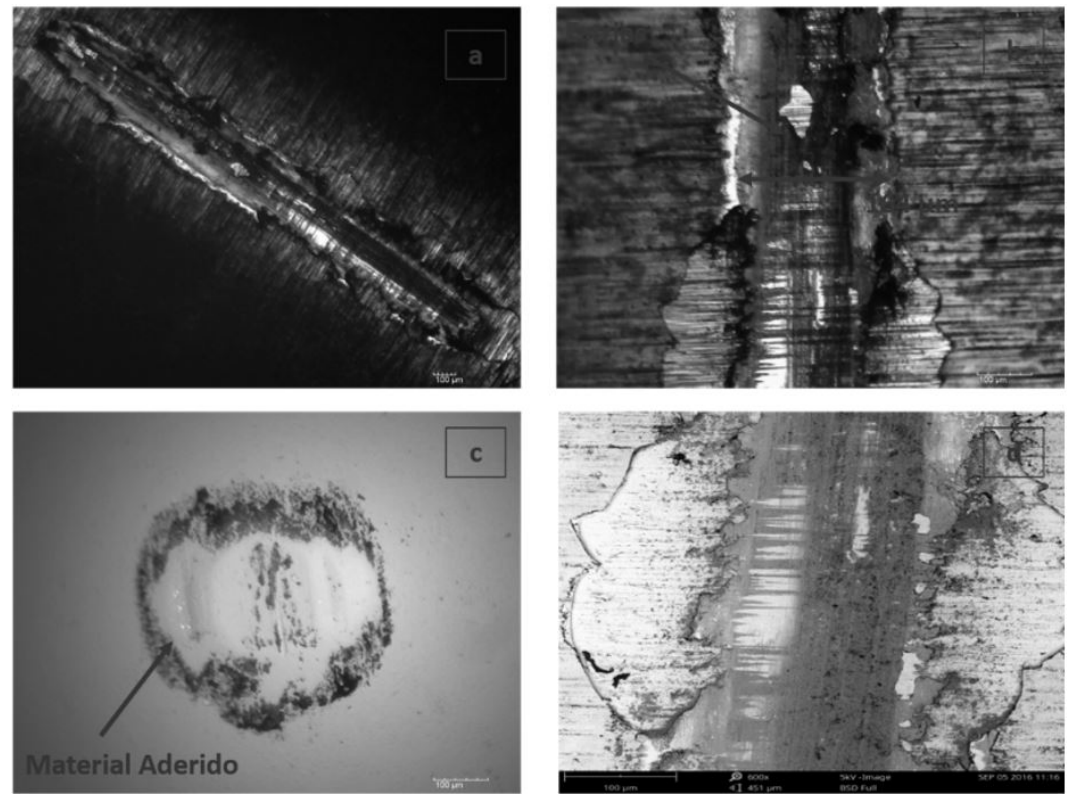

Figura 6. Trilhas de desgaste analisadas para a amostra EN 400-I: (a) Visão geral da trilha; (b) Medida da largura da trilha; (c) Análise da contra-face; (d) Imagem ampliada da trilha.

o revestimento, considerando que a difusão é acelerada em temperaturas elevadas.

Com relação ao mecanismo de desgaste, ficou claro após a análise das trilhas que o desgaste abrasivo é o principal elemento atuante, embora se encontre também mecanismo de desgaste adesivo. Isso é muito devido ao fato de haver partículas duras de $\mathrm{Ni}_{3} \mathrm{P}$ agindo como terceiro corpo no caso dos revestimentos com tratamento térmico, favorecendo a abrasão [26]. Para o caso do revestimento de Ni-P sem tratamento térmico, a explicação para uma fraca atuação do mecanismo adesivo seria pelo fato de existir uma grande diferença de durezas entre os materiais empregados ao longo do ensaio, visto que o fenômeno da adesão é mais frequentemente encontrado quando há o contato de materiais com durezas semelhantes [25].

Foi realizada uma análise via MEV/EDS em pontos dentro das trilhas após os testes de desgaste para a melhor condição verificada nos valores de COF (EN 320-9) e na pior condição $\left(\mathrm{EN} \mathrm{S} / \mathrm{T}^{\circ} \mathrm{T}^{\circ}\right)$. O resultado é bastante interessante (ver Figura 8), pois comprova que de fato a amostra com 

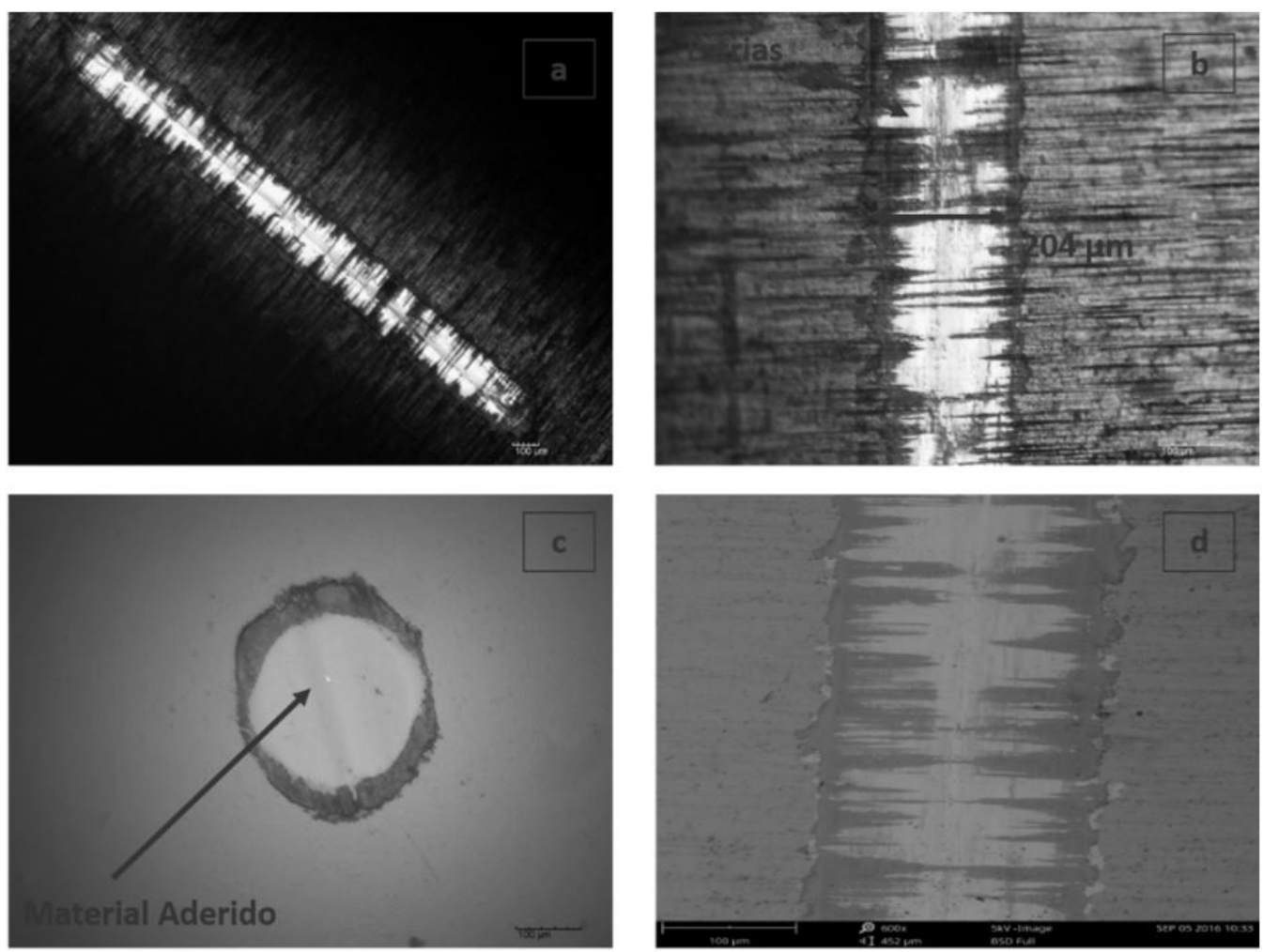

Figura 7. Trilhas de desgaste analisadas para a amostra EN 500-I: (a) Visão geral da trilha; (b) Medida da largura da trilha; (c) Análise da contra-face; (d) Imagem ampliada da trilha.

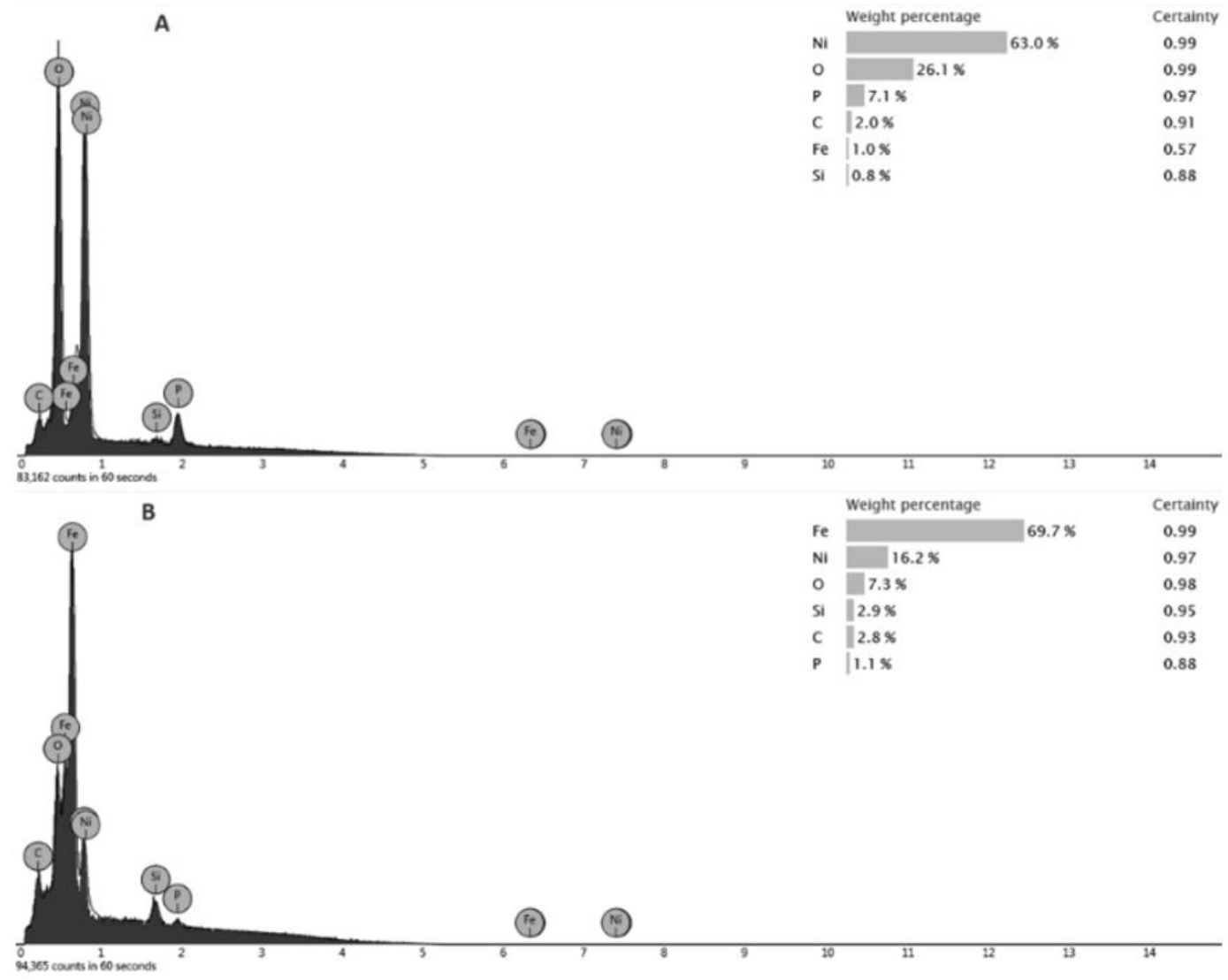

Figura 8. Espectrometria de energia dispersiva comparando as trilhas das amostras pós teste de desgaste: (A) EN 320-9; (B) EN S/Tํํ․ 
tratamento térmico em $320^{\circ} \mathrm{C}$ não alcança o substrato após o ensaio de desgaste, pois não fica evidenciado a presença do elemento ferro (elemento majoritário no aço inoxidável), a não ser em traços de $1 \%$ juntamente com o elemento silício. Em sentido oposto, a amostra de Ni-P sem tratamento térmico quebra o filme e mostra alto teor de ferro (em torno de 70\%).

\section{CONCLUSÕES}

Através dos ensaios realizados, pode-se concluir que revestimentos de Ni-P contendo aproximadamente $9 \%$ de fósforo em peso apresentam característica de material amorfo, e se tornam cristalinos após os tratamentos térmicos realizados neste trabalho em $320^{\circ} \mathrm{C}$ por 9 horas, $400^{\circ} \mathrm{C}$ por I hora e $500^{\circ} \mathrm{C}$ por I hora. A estrutura amorfa dá lugar a precipitação de cristalitos de $\mathrm{Ni}$ e de fosfeto de níquel $\left(\mathrm{Ni}_{3} \mathrm{P}\right)$ após aquecimento do depósito, fato este antecipado através do pico exotérmico obtido como resultado da análise de DSC.

A resistência ao desgaste nas condições apresentadas no teste é amplamente melhorada através de todos os tratamentos térmicos realizados nos revestimentos de Ni-P neste trabalho. As duas condições que apresentaram melhores resultados foram EN 320-9 e EN 400-I, provavelmente relacionadas a alteração microestrutural citada acima e pela formação de óxidos superficiais ocasionadas pelo tratamento térmico. Tanto o coeficiente de fricção é menor para estas duas amostras quanto $\circ$ tamanho da trilha de desgaste, $\circ$ que corresponde na prática a uma menor capacidade das amostras de sofrerem perda de material quando submetidas a movimento relativo com outro corpo com dureza elevada. A queda da resistência ao desgaste da amostra EN 500-I em relação aos demais tratamentos térmicos pode estar relacionada com o crescimento das fases presentes na sua microestrutura e pela formação de compostos intermetálicos difundidos do substrato em direção ao depósito.

Ainda foi verificado que os mecanismos de desgaste presentes nos depósitos foram tanto adesivo quanto abrasivo, este último em maior grau provavelmente devido aos compostos intermetálicos duros de $\mathrm{Ni}_{3} \mathrm{P}$ formados.

\section{Agradecimentos}

Aos laboratórios do Programa de Pós-Graduação em Engenharia de Minas, Metalúrgica e de Materiais (PPGE3M) da UFRGS: LACOR, LACER, LAMEF, LAPOL e LAPEC, e ao CNPq.

\section{REFERÊNCIAS}

I Balamurugan GM, Duraiselvam M, Anandakrishnan V. Comparison of high temperature wear behaviour of plasma sprayed WC-Co coated and hard chromium plated AISI 304 austenitic stainless steel. Materials \& Design. 2012;35:640-646.

2 El-Amoush AS, Abu-Rob A, Edwan H, Atrash K, Igab M. Tribological properties of hard chromium coated 1010 mild steel under different sliding distances. Solid State Sciences. 20I I; 13(3):529-533.

3 Leahey MH. Replacement of hard chrome electroplating by tungsten carbide based high velocity oxygen fueled thermal spray. New York: Rensselaer Polytechnic Institute; 2009 [acesso em 4 set. 2016]. Disponível em: http:// www.ewp.rpi.edu/ ernesto/SPR/Leahey-FinalReport.pdf

4 Apachitei I, Duszczyk J. Autocatalytic nickel coatings on aluminium with improved abrasive wear resistance. Surface and Coatings Technology. 2000; 132 (I):89-98.

5 Hamada AS, Sahu P, Porter DA. Indentation property and corrosion resistance of electroless nickel-phosphorus coatings deposited on austenitic high-Mn TWIP steel. Applied Surface Science. 2015;356: I-8.

6 Jiaqiang G, Yating W, Lei L, Bin S, Wenbin H. Crystallization temperature of amorphous electroless nickelphosphorus alloys. Materials Letters. 2005;59(13): 1665-1669.

7 Lonyuk B, Apachitei I, Duszczyk J. Effect of high-phosphorus electroless nickel coating on fatigue life of Al-Cu-MgFe-Ni alloy. Scripta Materialia. 2007;57(8):783-786.

8 Apachitei I, Tichelaar FD, Duszczyk J, Katgerman L. The effect of heat treatment on the structure and abrasive wear resistance of autocatalytic NiP and NiP-SiC coatings. Surface and Coatings Technology. 2002;149(2):263-278.

$9 \mathrm{Ma} \mathrm{C}$, Wu F, Ning Y, Xia F, Liu Y. Effect of heat treatment on structures and corrosion characteristics of electroless $\mathrm{Ni}-\mathrm{P}-\mathrm{SiC}$ nanocomposite coatings. Ceramics International. 20I4;40(7):9279-9284.

I0 Nava D, Davalos CE, Martinez-Hernandez A, Manriquez F, Meas Y, Ortega-Borges R, et al. Effects of heat treatment on the tribological and corrosion properties of electrodeposited Ni-P alloys. International Journal of Electrochemical Science. 2013;8:2670-268I.

I I Parente MMV, Mattos OR, Diaz SL, Lima P No, Miranda FF. Electrochemical characterization of Ni-P and Ni-Co-P amorphous alloy deposits obtained by electrodeposition. Journal of Applied Electrochemistry. 200 I;3 I(6):677-683. 
12 Szczygieł B, Turkiewicz A, Serafi czuk J. Surface morphology and structure of Ni-P, Ni-P-ZrO2, Ni-W-P, Ni-W-PZrO2 coatings deposited by electroless method. Surface and Coatings Technology. 2008;202(9): 1904-1910.

13 Taheri R. Evaluation of electroless nickel-phosphorus (EN) coatings. Saskatoon: University of Saskatchewan Saskatoon; 2002 [acesso em 4 set. 2016]. Disponível em: http://www.collectionscanada.gc.ca/obj/s4/f2/dsk3/SSU/ TC-SSU-02282003125442.pdf

14 Palaniappa M, Seshadri SK. Friction and wear behavior of electroless Ni-P and Ni-W-P alloy coatings. Wear. 2008;265(5-6):735-740.

I5 Sahoo P, Das SK. Tribology of electroless nickel coatings - A review. Materials \& Design. 20 I |;32(4): 1760- 1775.

16 Allen RM, VanderSande JB. The structure of electroless Ni P films as a function of composition. Scripta Metallurgica. 1982;16(10):1161-1164.

17 Mallory GO, Hajdu JB, editors. Electroless plating: fundamentals and applications. Orlando, Fla: AESF; 1990. 539 p.

18 Sribalaji M, Arunkumar P, Babu KS, Keshri AK. Crystallization mechanism and corrosion property of electroless nickel phosphorus coating during intermediate temperature oxidation. Applied Surface Science. 20 15;355:I I 2- 120.

19 Franco M, Sha W, Aldic G, Malinov S, Çimeno lu H. Effect of reinforcement and heat treatment on elevated temperature sliding of electroless $\mathrm{Ni}-\mathrm{P} / \mathrm{SiC}$ composite coatings. Tribology International. 2016;97:265-27I.

20 Bigdeli F, Allahkaram SR. An investigation on corrosion resistance of as-applied and heat treated Ni-P/nanoSiC coatings. Materials \& Design. 2009;30(10):4450-4453.

2I Rabizadeh T, Allahkaram SR, Zarebidaki A. An investigation on effects of heat treatment on corrosion properties of $\mathrm{Ni}-\mathrm{P}$ electroless nano-coatings. Materials \& Design. 2010;31(7):3174-3179.

22 Lee CK. Corrosion and wear-corrosion resistance properties of electroless Ni-P coatings on GFRP composite in wind turbine blades. Surface and Coatings Technology. 2008;202(19):4868-4874.

23 Vitry V, Delaunois F, Dumortier C. Mechanical properties and scratch test resistance of nickel-boron coated aluminium alloy after heat treatments. Surface and Coatings Technology. 2008;202(I4):3316-3324.

24 Palaniappa M, Seshadri SK. Friction and wear behavior of electroless Ni-P and Ni-W-P alloy coatings. Wear. 2008;265(5-6):735-740.

25 Menezes PL, Nosonovsky M, Ingole SP, Kailas SV, Lovell MR, editors. Tribology for scientists and engineers. New York: Springer; 2013 [acesso em em 4 set. 20I6]. Disponível em: http://link.springer.com/I0.1007/978-I-46I41945-7

26 Sadeghzadeh-Attar A. AyubiKia G, Ehteshamzadeh M. Improvement in tribological behavior of novel sol-enhanced electroless Ni-P-SiO2 nanocomposite coatings. Surface and Coatings Technology. 2016;307, Pt A, 837-848. https:// doi.org/10.1016/j.surfcoat.2016.10.026.

Recebido em: II Nov. 2016

Aceito em: 19 Dez. 2016 